
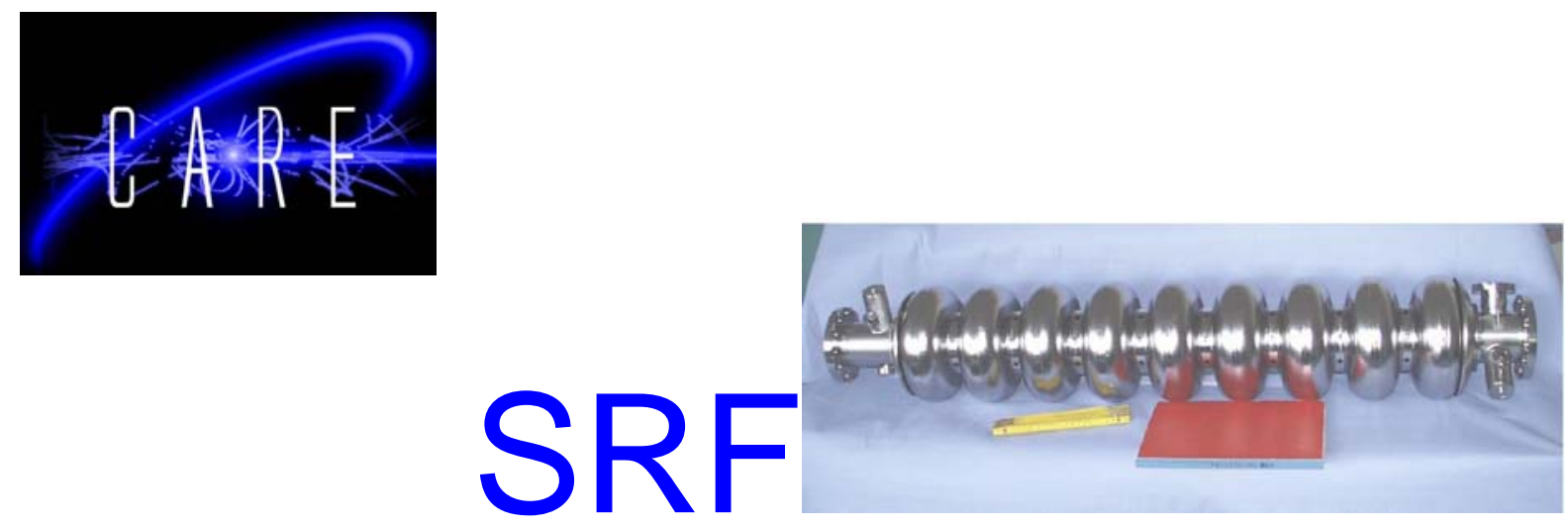

\title{
Improved Design of the ILC Blade-Tuner for Large Scale Production
}

\author{
Carlo Pagani, Angelo Bosotti, Nicola Panzeri
}

INFN Milano, Italy

\begin{abstract}
The ILC superconducting linacs ask for the use of a compact and cost effective tuner design with no interference with the cavity end group area. The integration of the piezo-assisted fast tuning option made the Blade-Tuner, successfully tested at DESY on the superstructures, the most viable candidate to be included into the ILC BCD. In the perspective of large scale production and on the basis of the experience acquired so far, two alternative prototypes have been recently designed and built. They mainly differ for the materials adopted (titanium or stainless steel) and have been optimized to minimize material and construction cost, while fulfilling the reviewed performances required for the high gradient cavity operation up to $35 \mathrm{MV} / \mathrm{m}$ or even higher. In this paper we discuss the rationales that brought us to the current solutions, together with a critical comparison of the two systems behaviour and cost.
\end{abstract}

Contribution to the " $22^{\text {nd }}$ Particle Accelerator Conference PAC07", Albuquerque (USA), 25-29 June 2007

Work supported by the European Community-Research Infrastructure Activity under the FP6 "Structuring the European Research Area" programme (CARE, contract number RII3-CT2003-506395) 


\title{
Improved design of the ILC Blade-Tuner for large scale production
}

\author{
Carlo Pagani, Angelo Bosotti, Nicola Panzeri INFN Milano, Italy
}

\section{Abstract}

The ILC superconducting linacs ask for the use of a compact and cost effective tuner design with no interference with the cavity end group area. The integration of the piezo-assisted fast tuning option made the Blade-Tuner, successfully tested at DESY on the superstructures, the most viable candidate to be included into the ILC BCD.

In the perspective of large scale production and on the basis of the experience acquired so far, two alternative prototypes have been recently designed and built. They mainly differ for the materials adopted (titanium or stainless steel) and have been optimized to minimize material and construction cost, while fulfilling the reviewed performances required for the high gradient cavity operation up to $35 \mathrm{MV} / \mathrm{m}$ or even higher.

In this paper we discuss the rationales that brought us to the current solutions, together with a critical comparison of the two systems behavior and cost.

\section{INTRODUCTION}

A new design of the blade tuner has been developed in order to optimize the total cost of the apparatus while improving their performances. This is well justified by the fact that more than 16000 coaxial tuners are required for the ILC project. The starting point of this final design was the existing blade tuner that proved to fulfil the slow tuner requirements $[1,2]$.

Two aspects have been considered in the optimization process: first of all a whole design refinement of rings and blades has been performed. This allowed to develop a unique geometrical solution that can be realized with the rings both in titanium or stainless steel. The second aspect considered concerns a major simplification of the driving mechanism and the moving of the motor from a central to a lateral position, thus freeing some space in correspondence of the invar rod of the ILC cryomodule.

Moreover, the adoption of a coaxial tuning solution allows to redesign the helium tank and its connection to the cavity, thus obtaining a more effective and cheaper solution.

\section{TUNER DESIGN}

A brief summary of the design guidelines and procedures followed is here reported.

The new tuner design has been driven by these considerations:

- the cost of materials (Ti) is steeply increasing;

- the cavity stability should have positive benefits from a lighter and more compact tuner design;
- the tuner strength and stiffness characteristics should be commensurate to the action on the helium tank and to the total stiffness of parts near the cavity.

- The original layout of the blade tuner [3] that assures a well symmetric behavior has been kept, but the main parameters that define the whole geometry of the rings, of the blades and of their distribution have been changed in order to achieve higher performances at a lower cost.

Keeping in mind these criteria and the necessity to have at least the same tuning capabilities of the previous blade tuner, different solutions have been analyzed. They mainly differ for the material considered and the geometry of the blades. A list of all the studied combinations is reported in [4].

\section{Optimal configuration}

Different finite element simulations have been performed in order to evaluate the tuner capabilities in different working conditions, and therefore to be able to chose the best performing geometry. An example of obtained results is pictured in figure 1, that report the von Mises stresses for the blade of configuration C (material titanium, new geometry). The values reported have been evaluated at the maximum admissible deformation.

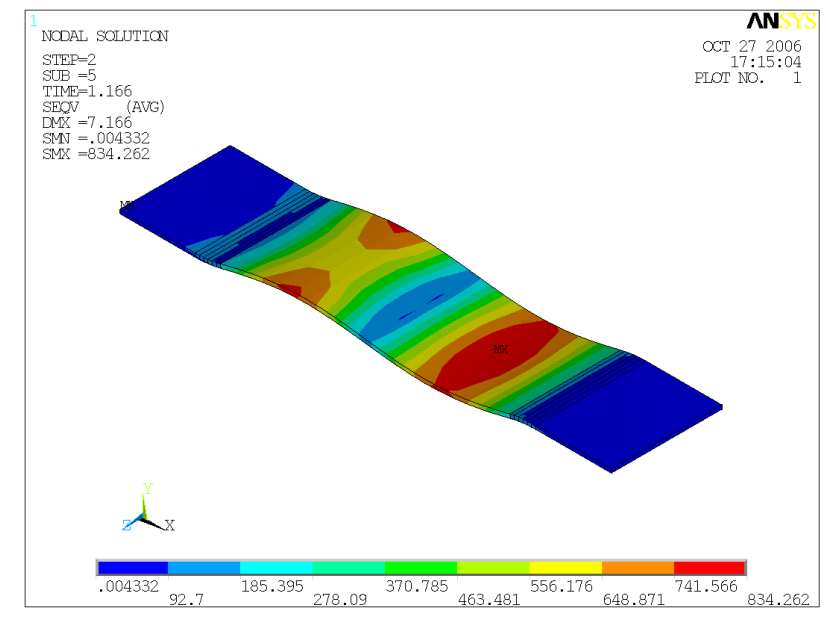

Figure 1: von Mises stresses (configuration C)

A summary of results for the chosen configurations is reported in table 1 . It contains the loads obtained from limit or buckling analysis, that have been used for the definition of the best blade configuration.

The numbers of required blades has been estimated by assuming a design force $F_{S}=4155 \mathrm{~N}$. This value is equal to the maximum compression load that can occur to the tuner for a pressure difference of 1 Bar between the inside and the outside of the helium tank. The buckling strength 
of blades has been reduced by a safety factor of 3 , while in the case of the limit load a safety factor of 1.5 has been assumed. The possible choices are reported in table 2 .

Because the final cost will depend from the machining operations, we plan to use a total number of $2 \times 48$ blades in configuration $\mathrm{C}$ or $\mathrm{F}$. They will be grouped in 6 packs of 4 blades each, for any of the half rings, thus allowing a
$75 \%$ reduction of welds respects to the original configuration A.

As a consequence of this choice we prefer to use the collinear blade position, and not the alternate one proposed in [7]. This will simplify the machining operations without a noticeable weight increase.

Table 1: summary of finite element results

\begin{tabular}{|c|c|c|c|c|}
\hline Combination & $\begin{array}{c}\text { Limit load in } \\
\text { stressed state (N) }\end{array}$ & $\begin{array}{c}\text { Max load without } \\
\text { plastic strains (N) }\end{array}$ & $\begin{array}{c}\text { Limit load in non- } \\
\text { stressed state (N) }\end{array}$ & $\begin{array}{c}\text { Buckling load in } \\
\text { undeformed state (N) }\end{array}$ \\
\hline C & 486 & 456 & 496 & 290 \\
\hline F & 824 & 693 & 804 & 519 \\
\hline
\end{tabular}

\section{A NEW DRIVING SYSTEM}

A simplified driving system has been designed, reducing drastically the number and complexity of parts of the previous solution. The new system (figure 3) is mainly composed of two monolithic parts supporting the motor and the nut. The axial movement of the nut is directly transferred to the rotational one by the central rings.

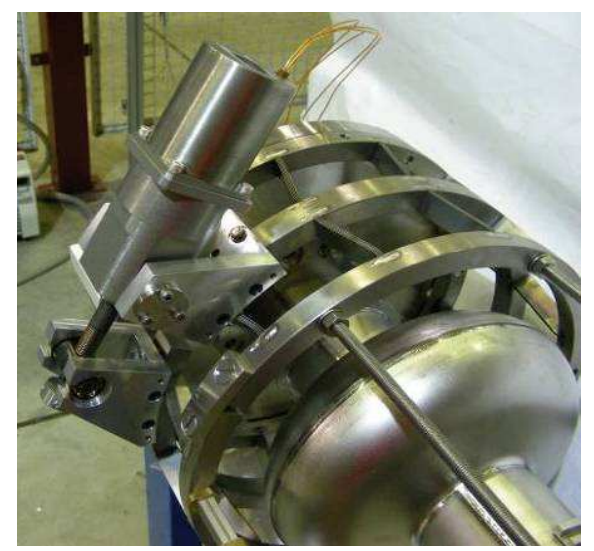

Figure 2: the optimized coaxial tuner for ILC

Although this arrangement simply pushes away the two central rings, therefore losing the complete symmetry, finite element computations and experimental tests proved the effectiveness of the solution [6].
Table 2: minimum blade requirements

\begin{tabular}{|c|c|c|c|c|}
\hline \multirow{2}{*}{$\begin{array}{c}\text { Blade } \\
\text { conf. }\end{array}$} & \multirow{2}{*}{$\begin{array}{c}\text { Adm. } \\
\text { blade } \\
\text { load }(\mathrm{N})\end{array}$} & \multirow{2}{*}{$\begin{array}{c}\text { Blade } \\
\text { stiffness } \\
(\mathrm{N} / \mathrm{mm})\end{array}$} & \multicolumn{2}{|c|}{$\begin{array}{c}\text { Minimum number of } \\
\text { blade on } 360^{\circ}\end{array}$} \\
\cline { 4 - 5 } & & strength & stiffness \\
\hline $\mathrm{C}$ & 162 & 5432 & 26 & 10 \\
\hline $\mathrm{F}$ & 268 & 10250 & 16 & 5 \\
\hline
\end{tabular}

\section{FURTHER IMPROVEMENTS}

The use of a coaxial tuner open different possibilities to optimize the geometry of the helium tank and of the cavity end group. In particular the tuner design just presented is lighter and more compact than the previous one. Positive consequences are a lower impact on the deflection of the cavity and a larger free space for the positioning of the cryomodule elements such as the invar rod. All this has been obtained maintaining the compatibility with the old design, so that this new version can be installed in the already made helium tank only by means of simple adapters. A comparison of the old and new tuner design is shown in figures 3 and 4 .

It is clear that the space in the upper part of the helium tank is almost all free, giving an easy access to the positioning of the invar rod.

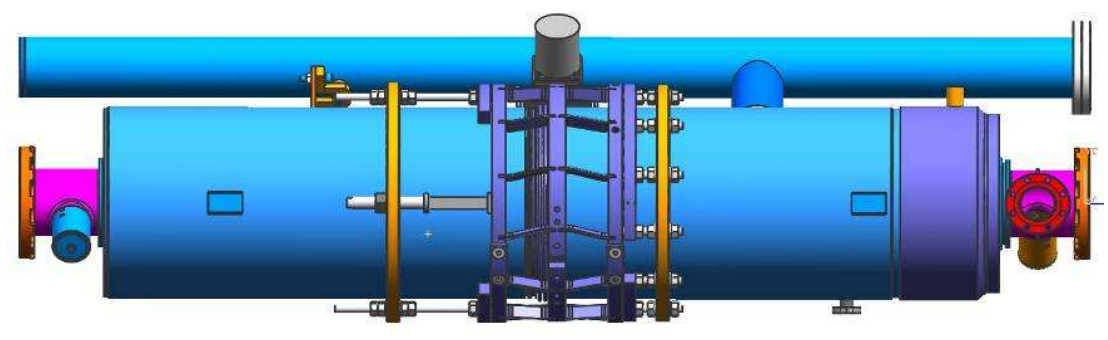

(a)

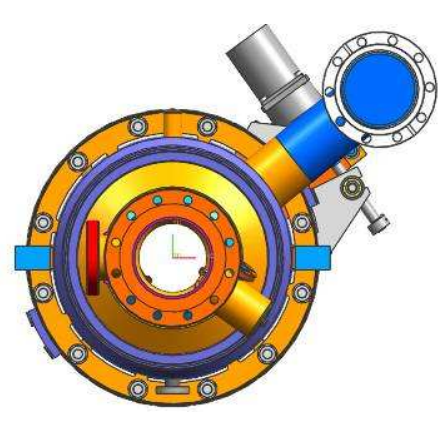

(b)

Figure 3: the new tuner installed on the old TTF helium tank. Lateral (a) and frontal (b) view. 


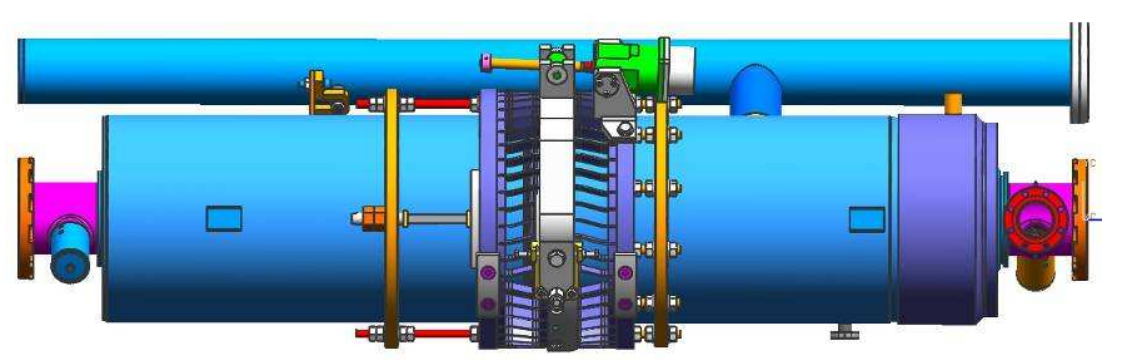

(a)

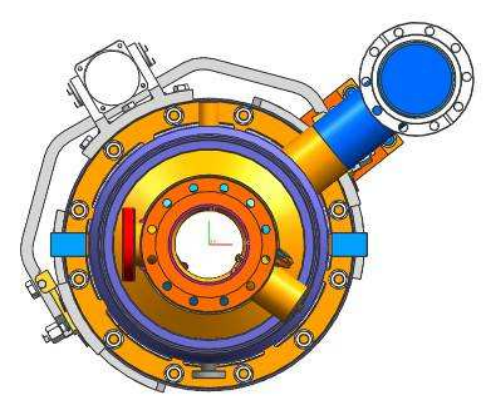

(b)

Figure 4: the old blade tuner installed on the TTF helium tank. Lateral (a) and frontal (b) view.

Moreover, the different position of the coaxial tuner with respect to the lateral one allows to modify the end group of the cavity, shortening it and changing its connection to the helium tank. In particular this should lead to a simpler solution, also more effective from the stiffness point of view. A preliminary solution aiming to rationalize the cavity end cones is shown in figure 5 . This design, originating from the solution adopted by the SNS and TRASCO cavities [5-6], is kept as simple as possible in order to reduce the overall cost and assembly operations.

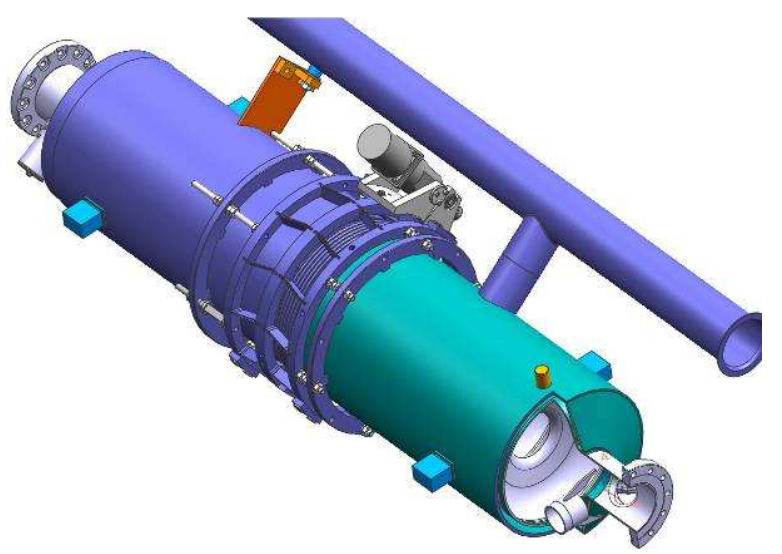

Figure 5: the tuner installed on a modified helium tank

As a plus, the axial stiffness is higher than that of the old solution, allowing the transmission of the $90 \%$ of the tuner displacements to the cavity, while in the old version the efficiency was only of $83 \%$. The design has to be fully defined, but this solution seems very promising in view of a large scale production of cavities for ILC.

\section{CONCLUSIONS}

The tuner design has been deeply revisited obtaining a much less expensive configuration and the possibility to make it both with titanium and stainless steel rings. This is an important capability in view of future developments concerning the use of stainless steel for the helium tank giving that the problem of the connection between titanium and stainless steel will be solved.
The new driving system solution is of course the simplest one possible, and it should also improve the overall tuner stiffness. Our characterization will be checked by several experimental tests whose execution at DESY is planned by the end of September. The test configuration is the one shown in figure 3 , where the titanium slim tuner is installed on a TTF helium tank.

As far as a stainless steel solution is not available for the helium tank, we decided to select the Ti version for the second cryomodule to be installed in the ILCTA at Fermilab. The rational of this choice is that our cost analysis for large scale production shows that the saving with SS is limited to a short 5\% while the driving motor is requested delivering twice the torque. As a conclusion the two versions, Ti and SS, will be both qualified, but the SS solution would be considered as a good alternative just in the case that a cheaper SS helium tank were available.

\section{ACKNOWLEDGEMENTS}

This activity is partially supported by the EC-Research Infrastructure Activity under the FP6 "Structuring the European Research Area" programme (CARE, contract number RII3-CT-2003-506395).

\section{REFERENCES}

[1] D. Barni, A. Bosotti, C. Pagani, R. Lange, H.B. Peters, "A New Tuner For TESLA", Proceedings of EPAC2002, Paris, France, p. 2205.

[2] J. Sekutowickz et al., "Test of Two Nb Superstructure Prototypes", in Phys. Rev. ST - AB, 7, 012002 (2004).

[3] C. Pagani et al., CARE-Note-2005-021-SRF.

[4] C. Pagani, N. Panzeri, "Comparative analysis of blade tuner optimization options for the ILC", Proceedings of APAC'07, Indore, India 29 January 30 February 2007, WEPMA123

[5] A. Bosotti et al., "Report on cavity A (TRASCO Z502) fabrication and test", CARE-Note-2005-001HIPPI.

[6] G. Ciovati et al., "Superconducting prototipe cavities for the spallation neutron source (SNS) project", Proceedings of PAC2001, Chicago, USA, p. 484. 